\title{
Creating the Future Test Range Infrastructure: Vision for a Wireless Inter-Range Network Environment
}

\author{
Thomas O O'Brien \\ U.S. Department of Defense Test Resource Management Center, 4800 Mark Center Dr Suite 07J22, \\ Arlington, VA 22350
}

\begin{abstract}
:
Radio frequency spectrum is a vital resource to Test \& Evaluation (T\&E) and is used to support nearly every test in every environment, yet the availability of RF spectrum to support testing has diminished since the 1990s. A major testing challenge is balancing the development of complex systems requiring the transmission of increasingly large amounts of data with a diminished amount of spectrum available to test facilities and ranges. This inverse relationship creates spectrum congestion at test facilities and can delay test activities.
\end{abstract}

In order to continue testing systems efficiently and adequately, a major test infrastructure paradigm shift is being developed looking toward a bi-directional, highly integrated, wireless, inter-range network environment that seamlessly supports any and all range operations and data types with even greater spectrum efficiency than currently possible. The implementation of a wireless, inter-range network environment relies on leveraging current efforts to develop a network-based telemetry capability and applying a mobile wireless "cellular" paradigm. While the T\&E environment differs from the mobile wireless environment, adaptation of certain wireless technologies to the testing environment might provide significant benefits that satisfy increasing testing requirements.

This paper highlights the spectrum availability issues facing the major Department of Defense test ranges and the vision developed by the Department of Defense Test Resource Management Center to implement a wireless inter-range network environment to support test data transmission requirements. The ultimate goal is to garner support and awareness for the effort both domestically and abroad and show U.S. commitment to invest in spectrally efficient technologies, methodologies, and paradigms to support data transmission requirements.

Key words: Telemetry, Test and Evaluation, Test Infrastructure, Network Environment, Network Based Telemetry, Test Resource Management Center

\section{Introduction}

Radio frequency (RF) spectrum is a vital resource to the Test and Evaluation (T\&E) community and is used to support nearly every test in every environment, yet its availability to support these tests has been decreasing. Beginning in the early 1990s, the spectrum available to the Major Range and Test Facility Base (MRTFB) has significantly decreased. The widespread development and use of wireless communications devices (WiFi- and WiMAXenabled), as well as cellular and satellite services, has led to commercial entities seeking increasing amounts of spectrum to support their communication services. Spectrum bands affected domestically in the U.S. are typically those used for T\&E purposes, such as flight test telemetry (e.g., reallocation of the $1710-1755 \mathrm{MHz}, 1755-1780 \mathrm{MHz}, \& 2310-2360 \mathrm{MHz}$ bands).

Spectrum access is further complicated by increasing demand from other DoD users to support new or modified military systems. These complex military systems require the telemetering of a large amount of data to effectively characterize system performance during testing-an exponential growth in data transmission requirements over the past twenty-five years. RF spectrum availability affects test programs in many regards, most importantly cost and schedule. Insufficient access to RF spectrum leads to test scheduling delays which in turn lead to increased program costs.

To address the inverse relationship between decreasing spectrum availability and increased telemetry data requirements, the DoD Test Resource Management Center (TRMC) has developed a vision for a wireless, highly integrated, network to support all range data transmission in the most spectrally efficient manner possible. This vision represent a major shift in the test infrastructure paradigm, moving from a point to point link in which an omnidirectional antenna or wrap around antenna on the SUT is used 
to broadcast telemetry data to one or more parabolic, mechanically steered tracking antennas on the ground, each of which are only capable of tracking one test article at a time. In order to build towards this vision for test range infrastructure, the TRMC established several efforts to address spectrum resource issues. These technology development efforts focus on achieving greater efficiencies in the use of the available spectrum, pursuing additional spectrum in different frequency bands, implementing a robust networked telemetry capability, and leveraging cellular based technologies to support telemetry operations. For example, TRMC sponsored programs including Integrated Network Enhanced Telemetry (iNET), Cellular Based Range Telemetry (CRTM), Cellular Range Telemetry Network (CeRTN), and Flightline Radio Network (FRN) to build towards this vision and better use the spectrum that is available for T\&E.

\section{Spectrum Availability}

Spectrum is a limited commodity, just like oil, and its availability to the MRTFBs for testing has been constantly decreasing. In recent years, a disturbing trend has surfaced-increasingly complex systems requiring a large amount of data to be transmitted are being developed while the amount of spectrum available to the test range has been steadily decreasing-making it very difficult for test ranges to operate (Figure 1).

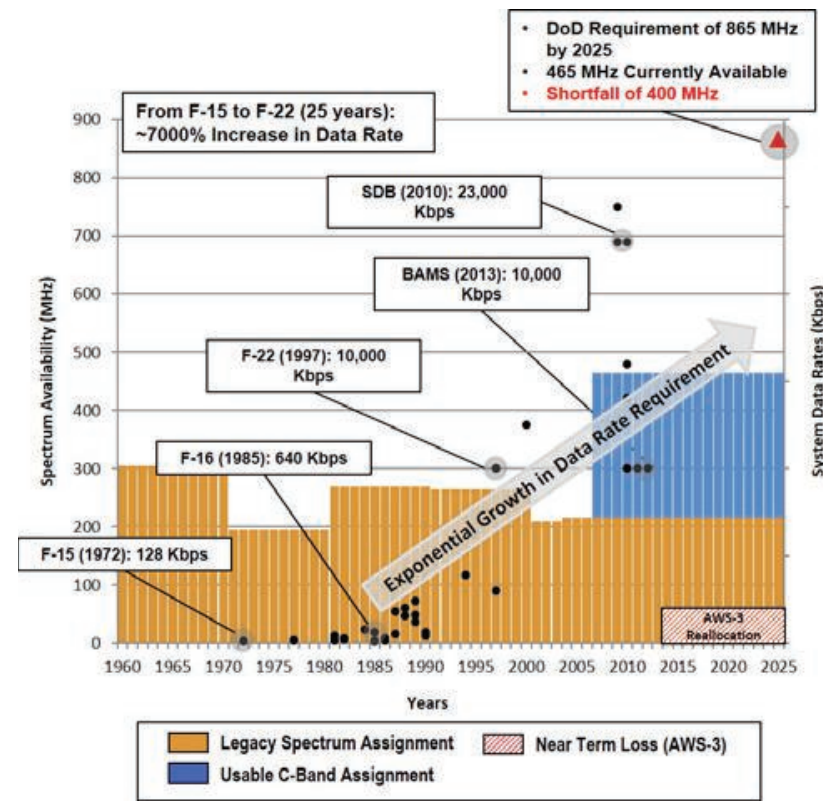

Figure 1- Spectrum \& Data Rate Trends

Domestic repurposing of RF spectrum in the U.S. has drastically changed the amount of spectrum that is available for testing. Figure 2 shows the historic allocations given the U.S. test ranges versus the RF spectrum currently available for testing.

\begin{tabular}{|c|c|}
\hline $\begin{array}{c}\text { Historic Frequency } \\
\text { Allocations, MHz }\end{array}$ & $\begin{array}{c}\text { Current Frequency } \\
\text { Allocations, MHz }\end{array}$ \\
\hline Lower L Band (TSPI) & $1350-1390$ \\
\hline
\end{tabular}

\begin{tabular}{|c|c|}
\hline $1350-1400$ \& 1427-1436 & \\
\hline $\begin{array}{c}\text { Lower L Band } \\
1435-1535\end{array}$ & $1435-1525$ \\
\hline $\begin{array}{c}\text { Shared Upper L Band } \\
1710-1850\end{array}$ & $1780-1850$ \\
\hline $\begin{array}{c}\text { Lower S Band (UAS) } \\
2200-2300\end{array}$ & $2200-2290$ \\
\hline $\begin{array}{c}\text { Upper S Band } \\
2310-2395\end{array}$ & $\begin{array}{c}\text { Spectrum Lost : } \\
\mathbf{1 5 9} \text { MHz ( 33\%) }\end{array}$ \\
\hline Sum : 484 MHz
\end{tabular}

Figure 2- Spectrum Allocations

This chart shows the effects that spectrum repurposing activities has on the testing environment- $33 \%$ of the spectrum that was once allocated for testing has been re-allocated to support other uses.

The loss of spectrum, increasing demand for spectrum to test complex systems, and the threat of future losses of spectrum have snowballed into a larger problem for test ranges-band congestion. The issue that has arisen is that as the amount of spectrum decreases, scheduling becomes more and more difficult and the telemetry bands are becoming heavily congested. As a result, it is estimated that $17 \%$ of all scheduling requests are denied due to insufficient spectrum as a result of band congestion ${ }^{1}$. Spectrum availability, and the prospect of future limitations on spectrum access are a driving factor towards a bi-directional wireless inter-range network environment.

\section{Current Range Telemetry Paradigm}

The test infrastructure at MRTFB sites is currently configured to support unidirectional point to point link in which an omnidirectional antenna (or wrap around antenna on a missile) on the test article is used to broadcast telemetry data to one or more parabolic, mechanically steered tracking antennas on the ground which are only capable of tracking one test article at a time. While this approach has traditionally supported telemetry operations, a new approach is necessary to adapt to the complex spectrum environment created by the growth in personal wireless devices and commercial cellular equipment. Furthermore, the current telemetry paradigm limits the spectral efficiencies that can be gained when compared to a bi-directional networked telemetry approach.

Shortfalls with the current test range telemetry paradigm include ${ }^{2}$ :

\footnotetext{
${ }^{1}$ Information taken from the Sarnoff "RDT\&E Spectrum Requirements Assessment", 5 August 2004; Estimate of the shortfall is for the Western Range facilities

2 Young, Tom and Radke, Mark, "An Innovative Approach to Modernizing Telemetry" ITEA Journal, 2016
} 


\section{- $\quad$ Limited flexibility in transmitted data format}

Currently, test data is acquired by the instrumentation system on the test article and encoded into formats that are pre-defined and referred to as a "data package". These data packages are composed of fixed length frames containing data along with synchronization words to delineate the beginning of each frame. These frames are then transmitted over a fixed bandwidth, fixed frequency (both configurable, but not changed during a test) from an omnidirectional blade antenna (or wrap around antenna on a missile) to a parabolic, mechanically steered tracking antenna at one or more ground stations.

\section{- Transmission of less urgent test data}

Often when conducting test missions, a series of "test cards" are created to test specific aspects of the aircraft during that flight. Test cards are generally conducted with the same data package that includes all parameters needed to be monitored for the entire mission, or a particular aspect of the current test phase.

\section{Allocation of infrastructure resources}

During a test mission, ground station resources are allocated to receive the telemetry signal and relay it to the mission control room. Since parabolic antennas only track one target at a time, they have to be dedicated to a test article for the duration of a mission. These telemetry ground stations (GS) are expensive to construct and maintain, most requiring to be manned during missions.

\section{- $\quad$ Non-optimized spectrum scheduling and usage}

Spectrum assignments for test operations are planned days in advanced to allow sufficient time for manual coordination and de-confliction. There are tools available to visualize spectrum occupancy and conflicts, but resolution of conflicts is a manually intensive process. Since the telemetry links can operate at distance of up to 150 nautical miles, frequencies are typically not re-used in instances where there is the potential for interference. This scheduling paradigm often requires frequencies for a single mission to be allocated across multiple ranges to prevent interference due to the power of the transmitter and gain of the ground antennas. The scheduling of frequencies across test ranges and facilities does not allow test planning tools to employ spatial diversity to reduce the probability of interference from adjacent signals and provide frequency re-use.

The TRMC is developing the core set of networked telemetry capabilities, including implementing a bidirectional telemetry link, as part of the Integrated Network Enhanced Telemetry (iNET) project. These capabilities are enabling components for the Wireless Inter-range Network Environment. The iNET program is developing a new telemetry system, employing a network architecture for airborne platforms that will replace the current instrumentation systems with industry standard Ethernet and add a bidirectional network channel from the aircraft to the ground. iNET project status and changes to the current telemetry paradigm are found in the conference proceedings as part of the technical paper titled "iNET: Impacts to the Telemetry Community".

\section{Wireless Inter-Range Network Environment}

The implementation of a wireless, inter-range network environment focuses on leveraging current efforts to develop a network based telemetry capability and mobile wireless "cellular" technologies. The wireless, cellular approach has been in use since the 1980s for commercial applications and has evolved and improved to the point where wireless voice and data services have become ubiquitous allowing instant communication. Commercial wireless carriers have challenges that are similar to those experienced by the MRTFB facilities and ranges, specifically how to cost effectively support increasing numbers of high bandwidth users over large geographical areas with a limited amount of spectrum. While the T\&E environment differs from the mobile wireless environment, adaptation of current wireless technologies to this environment provides significant benefits and efficiencies.

Shifting the focus from the use of point to point links to a networked architecture will require changes in the terrestrial range infrastructure-specifically moving from parabolic, mechanically steered tracking antennas to an arrangement of smaller less network enabled ground stations which could be deployed across a range to provide the necessary coverage to flight line areas, hangars, and flight areas. To accommodate the existing range infrastructure, current long range antenna systems could be modified to provide additional coverage over ocean ranges and large expanses of open terrain where it might be impractical to deploy cellular based ground stations.

For example, in a wireless, inter-range network environment, multiple ground stations arranged in a manner similar to Figure 3 that cover a majority of range areas and provide additional capacity based on frequency re-use. In this scenario, these ground stations would be connected with either a wireless point to point, wireless mesh network, or fiber backhaul to transport data back to the mission control room. Each ground station would have a smaller coverage area to maximize spectrum re-use.

The test article transmit power can be automatically controlled to provide the optimum power required to maintain a stable link while minimizing interference to adjacent ground stations. When additional coverage is needed in a remote location, a mobile ground station could be deployed to provide the needed coverage. Each color in Figure 3 denotes a different frequency range in which a ground station would operate. 

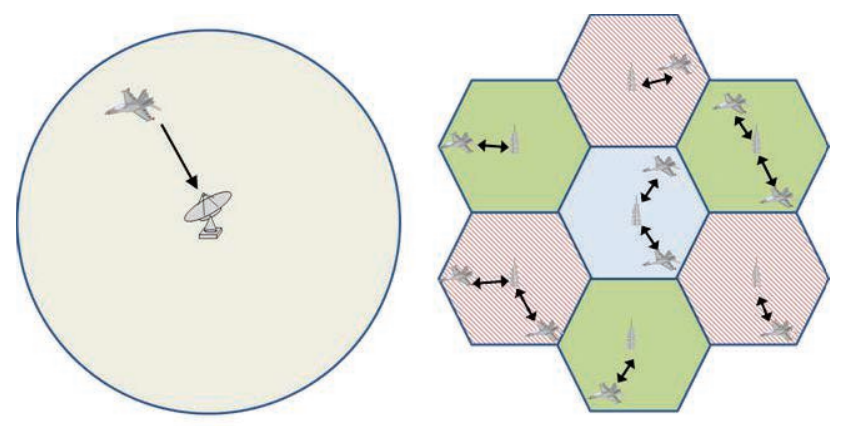

Figure 3- Example of Traditional data transmission method versus a Cellular Based Approach

Additionally, the commercial wireless industry invested heavily in developing various data modulation/transmission schemes to enable access to wireless channels by multiple users. Each of these schemes have benefits and shortcomings, and all would have to be adapted to the test range environment (e.g. increased Doppler shift due to higher velocities, different operating frequencies). The T\&E community has invested in the technology to leverage one such modulation scheme, Orthogonal Frequency Division Multiple Access (OFDMA). In addition to OFDMA, other industry developed multiple access schemes like Single Carrier Frequency Division Multiple Access (SCFDMA), which is being employed in the latest wireless standard of Long Term Evolution (LTE), could be leveraged and are typically more suitable for channels with high speed users. Additionally, different access schemes may prove more appropriate for use on the uplink portion of the communication channel as opposed to the downlink. When using OFDMA as a multiple access scheme, each test article would be allocated a set of subcarriers, enabling each ground station to support multiple test articles in each "cell". Supporting multiple simultaneous users with OFDMA will also save spectrum that would normally be lost to guards bands between single carrier signals. When channel conditions are suitable, more advanced modulations (e.g., SOQPSK-TG, ARTM-CPM, QAM16, QAM64) could be used to modulate the subcarriers providing even more throughput per unit of spectrum.

As a test article moves from one ground station to another, the network infrastructure system would need to execute a "handoff" of the test article to maintain connectivity. These handoff mechanisms would also be leveraged from the mobile wireless industry, which seamlessly allow movement from one cell to another while maintaining both voice and network connectivity. In addition to handing over data channels from cell to cell, additional handover mechanisms at the network level would need to be employed. As a test article moves to another range's network, it registers with a home agent on its home network and provides a "care of address".

In terms of resource scheduling, ground stations and spectrum that are required to support a test mission are scheduled days in advance. This is mainly due to the limited number of ground stations (which only supports a single test article each) on a range, as well as the manually intensive process of frequency de-confliction often requiring cooperation between several ranges. By using a cellular-based approach, frequency assignments and de-confliction is done once during the planning and deployment phase of the ground stations or when modifications are required (ground stations added or removed). Ground station scheduling is also eliminated because each ground station can support multiple test articles simultaneously.

The TRMC is sponsoring several efforts to leverage, develop, or modify commercial cellular technologies to operate in the testing environment to support telemetry. The first such effort, the Flightline Radio Network with Seamless handoff (FRNSH) effort, is leveraging commercially available cellular technologies operating in the 1710-1755 MHz 3GPP band to support pre-flight test telemetry requirements and instrumentation check out in the hangar, flightline, and taxiway environments. Currently, AMT systems are turned on pre-test when an aircraft is in the hangar or flightline to ensure that onboard instrumentation systems are functioning properly. Unfortunately, this situation uses RF spectrum allocated for flight testing and consumes spectrum that could otherwise be used to support an additional flight test mission. By shifting this pre-test instrumentation telemetry requirement onto non-AMT spectrum, such LTE or 3GPP commercial spectrum, an additional flight test mission can be conducted using the AMT dedicated spectrum. Additionally, the Cellular Range Telemetry (CRTM) and Cellular Range Telemetry Network (CeRTN) efforts are modifying commercial cellular components, such as eNodeB and user equipment, to operate in portions of spectrum allocated for AMT in the U.S. These efforts are developing the initial ground infrastructure required to implement a wireless inter-range network environment and are investigating technical components of the future vision for a test range infrastructure such as Doppler effects using cellular ground stations and cellular handover mechanisms and techniques. These technology development and demonstration efforts will lay the ground work to build on the CTEIP iNET project towards a wireless interrange network environment to support all range data transmission requirements in the most spectrally efficient manner possible.

\section{Conclusion}

In order to fully realize a robust a wireless range network, continued investment in spectrally efficient technologies will be required. There are several thrusts within the DoD to invest in technologies that support more efficient usage of the RF spectrum and enable spectrum sharing opportunities.

Once the iNET project is completed and delivers the core networked telemetry components and the technology demonstrations and development efforts from the FRNSH, CRTM, and CeRTN projects are completed, the TRMC, in conjunction with the MRTFB facilities and ranges, will need to explore future investments to optimize cellular technologies 
for use in the testing environment. Once the appropriate investments are made to tailor the cellular technologies to the testing environment, budgetary decisions can be made to fully implement a cellular telemetry capability across the MRTFB.

\section{References}

[1] Sarnoff Corporation: "RDT\&E Spectrum Requirements Assessment”, 2004.

[2] Young, Tom and Radke, Mark, "An Innovative Approach to Modernizing Telemetry" ITEA Journal, 2016.

\section{Glossary}

AFB: Air Force Base

AMT : Aeronautical Mobile Telemetry

CTEIP: Central Test and Evaluation Investment

Program

DoD: U.S. Department of Defense

iNET: Integrated, Network Enhanced Telemetry

MRTFB: Major Range and Test Facility Base

SUT: System Under Test

T\&E: Test and Evaluation

TRMC: Test Resource Management Center 